\title{
CT and MRI Features of a Solid Hemangioblastoma of Cerebellar Vermis in a Child: A Case Report
}

\author{
Ling Sun ${ }^{1}$ Heng Liu ${ }^{2}$ Wuchao Li ${ }^{2}$ Lin Jiang ${ }^{2}$ Chong Tian² Xinyu Wang ${ }^{2}$ Yihua Zhou ${ }^{3}$ \\ Tijiang Zhang ${ }^{2}$ Qiyong Gong ${ }^{1}$
}

${ }^{1}$ Department of Radiology, West China Hospital of Sichuan University, Chengdu, China

2 Department of Radiology, Affiliated Hospital of Zunyi Medical

College, Zunyi, China

${ }^{3}$ Department of Radiology, Saint Louis University School of Medicine, Saint Louis, Missouri, United States

J Pediatr Neuroradiol 2015;4:19-24.

Address for correspondence Tijiang Zhang, MD, Department of Radiology, Affiliated Hospital of Zunyi Medical College, Zunyi, China (e-mail: tijzhang@163.com).

\begin{abstract}
Keywords

- hemangioblastoma

- computed tomography

- magnetic resonance imaging

- posterior fossa tumor

Solid hemangioblastomas of cerebellum are extremely rare in children and may be misdiagnosed as other pediatric intracranial tumors. A 13-year-old girl presented with a 5-day history of cerebellar dysfunction without apparent inducement. A computed tomography scan revealed a well-defined solid isodense lesion in the inferior aspect of the cerebellar vermis with intense homogeneous enhancement. Magnetic resonance imaging demonstrated a well-circumscribed solidly enhancing mass with serpentine signal voids in the periphery. Histopathology revealed a hemangioblastoma after surgical excision. This case draws our attention to a differential diagnosis of hemangioblastomas in children when a solid tumor is found in the cerebellum with hypervascularity and intense enhancement.
\end{abstract}

\section{Introduction}

Hemangioblastomas are benign World Health Organization (WHO) grade I tumors that have been classified under the category of other neoplasms related to the meninges in the fourth edition of WHO classification of the central nervous system (CNS) tumors with an uncertain origin. ${ }^{1}$ They account for 1.5 to $2.5 \%$ of all intracranial neoplasms and 7 to $12 \%$ of posterior fossa tumors in adults, with $85 \%$ occurring in the cerebellum, including the cerebellar hemispheres and the vermis. They may occur either in sporadic forms or in association with von Hippel-Lindau (VHL) syndrome, to which only 5 to $30 \%$ of all cerebellar hemangioblastomas are attributed. ${ }^{2}$ Most hemangioblastomas occur in young adults of 20 to 40 years of age. However, in children, these tumors are extremely rare, with an incidence of less than 1 per million and accounting for much less than $0.5 \%$ of all pediatric brain tumors. ${ }^{3}$ In this report, we present a rare case of a 13-year-old girl with solid hemangioblastoma in cerebellar vermis without VHL syndrome.

\section{Case Report}

A 13-year-old girl presented with a 5-day history of vertigo, headache, nausea, vomiting, and loss of balance without apparent inducement. Physical examination revealed gait ataxia with consciousness. She had weakness in the limbs of power grade III. The physiological reflexes were normal and the pathological reflexes were negative. Abdominal screening by ultrasonography revealed no evidence of primary or metastatic neoplasm or cysts. The patient did not have vision change and the eye examination was normal. There was no family history of VHL syndrome.

A computed tomography (CT) scan revealed a solid isodense lesion with CT value of $44 \mathrm{HU}$ in the inferior cerebellar vermis received

June 26, 2013

accepted after revision

January 15, 2015

published online

October 6, 2015
Copyright @ 2015 by Georg Thieme

Verlag KG, Stuttgart • New York
DOI http://dx.doi.org/ 10.1055/s-0035-1564662. ISSN 1309-6680. 
(- Fig. 1A). The lesion was well defined and measured approximately $27 \mathrm{~mm} \times 19 \mathrm{~mm} \times 18 \mathrm{~mm}$ with intense homogeneous enhancement ( - Fig. 1B). The fourth ventricle and supratentorial ventricles were enlarged, indicating hydrocephalus due to outlet obstruction of the fourth ventricle. Computed tomography angiography (CTA) showed that the lesion was associated with enlarged feeding arteries originating from the right posterior inferior cerebellar artery (-Fig. 1C and D).

Magnetic resonance imaging (MRI) demonstrated a well-circumscribed oval mass in the inferior cerebellar vermis, measuring $28 \mathrm{~mm} \times 25 \mathrm{~mm} \times 19 \mathrm{~mm}$. The lesion was isointense on T1-weighted images and hyperintense on T2-weighted and fluid-attenuated inversion recovery (FLAIR) images (-Fig. 2A-D). The internal and peripheral serpentine signal voids were seen on T2-weighted and FLAIR images, suggestive of hypervascularity. After the administration of gadolinium-based contrast medium, the lesion showed prominent enhancement (-Fig. 2E-F). Mass effect with obstructive hydrocephalus was evident.

The patient underwent a suboccipital craniotomy for resection of the tumor. AY-shaped dural incision was created to access the mass. There was an increased size of the posterior fossa as well as the intracranial pressure. The tumor was located in the inferior cerebellar vermis, protruding into the inferior aspect of the fourth ventricle, and it was closely adhered to the medulla oblongata. The fourth ventricle was enlarged and deformed, accounting for the apparent cystic component seen on the preoperative images. There was no tumor capsule or a clear margin with the surrounding tissues. The tumor was red, fleshy, and extremely vascular with multiple prominent feeding arteries and draining veins which were located in the back of the tumor. Finally, a gross total resection of the tumor was achieved under microscope. The tumor was solid without cystic content, measuring $\sim 30 \mathrm{~mm} \times 30 \mathrm{~mm} \times 30 \mathrm{~mm}$.

Histological examination of the tissue revealed proliferating blood vessels with stromal cells which were medium in size and characterized by large nuclei and abundant partly vacuolated cytoplasm. No atypical cells were seen ( - Fig. 3A). Immunohistochemical staining showed that blood vessel endothelium was positive for CD31 and CD34 (-Fig. 3B). The stromal cells were immunopositive for glial fibrillary acidic protein and neuron-specific enolase. These histopathological findings were consistent with a diagnosis of hemangioblastoma (WHO grade I).

The postoperative MRI of the brain in 1 week revealed a complete gross resection without any residual tumor

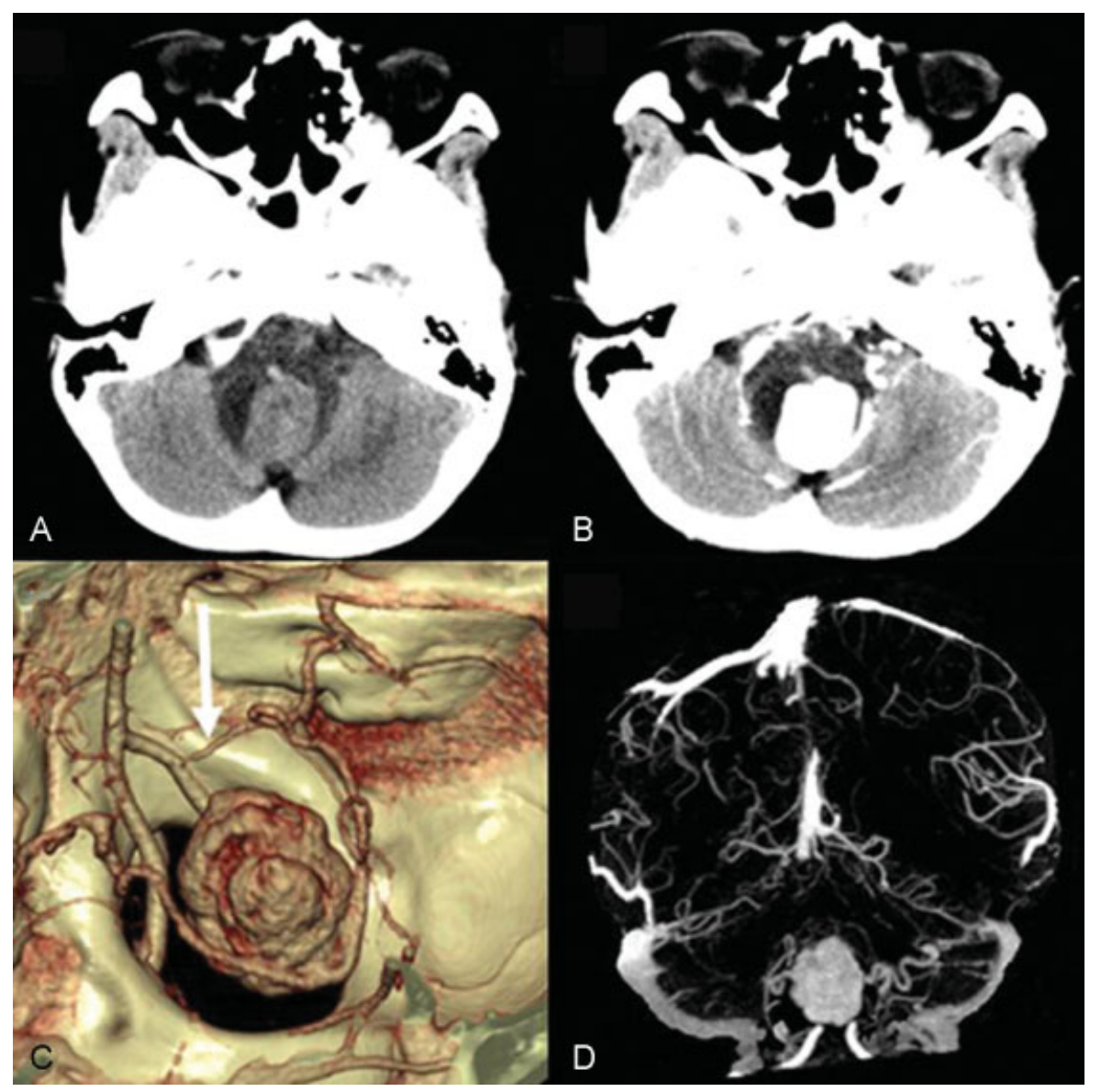

Fig. 1 CT scan images of the brain showing a solid hemangioblastoma in the inferior cerebellar vermis. (A) An axial unenhanced CT image shows a well-defined, isodense, solid lesion in the inferior cerebellar vermis. (B) An axial enhanced CT image demonstrates intense homogeneous enhancement of the mass. (C) A CTA volume-rendered image and (D) maximum intensity projection show the lesion with enlarged feeding arteries, with the white arrow pointing at the right posterior inferior cerebellar artery. 


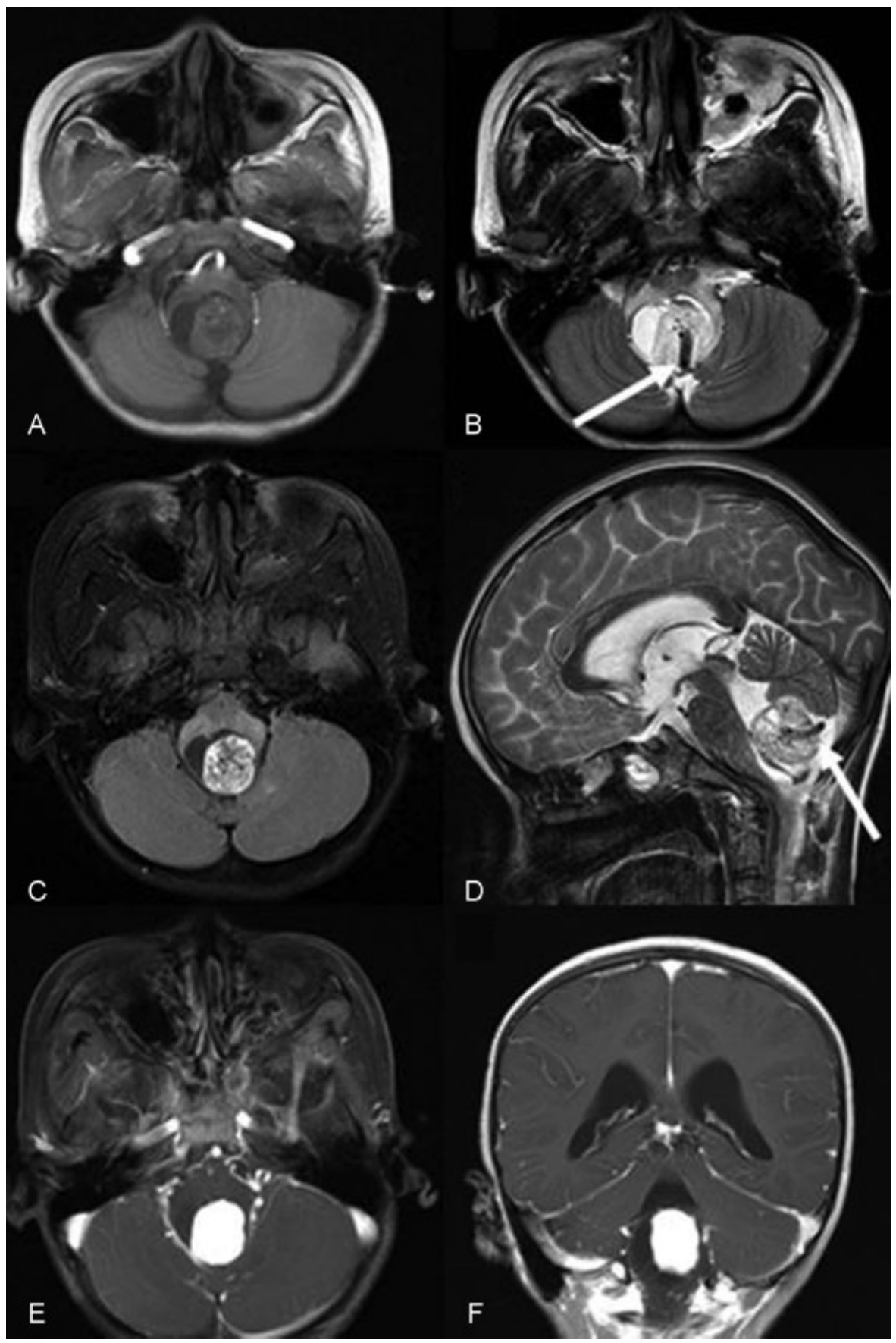

Fig. 2 MR images of the brain showing a solid hemangioblastoma in the inferior cerebellar vermis. (A) An axial T1-weighted image shows a wellcircumscribed isointense solid lesion in the inferior cerebellar vermis. (B) An axial T2-weighted image, (C) an axial FLAIR image, and (D) a sagittal T2-weighted image show a hyperintense lesion with internal and peripheral serpentine signal voids on precontrast MR images (arrows pointing at the signal voids). (E) An axial gadolinium-enhanced image and $(F)$ a coronal gadolinium-enhanced image show intense enhancement. Note the presence of obstructive hydrocephalus.

( - Fig. 4A-D). The clinical symptoms of the patient had resolved at 1-year follow-up.

\section{Discussion}

In this report, we presented the imaging features of an extremely rare tumor in the young pediatric population that could be mistaken for other pediatric intracranial tumors.
Hemangioblastomas are uncommon tumors of young adults, but unlike in patients with sporadic disease, when associated with VHL syndrome, they may occur at a younger age. ${ }^{2}$ Although cases of hemangioblastomas are reported in children, these are relatively uncommon. ${ }^{4,5}$ Unfortunately, no details of the imaging features were described in these cases. As a result, hemangioblastomas may not be initially considered in children presenting with CNS tumors. 


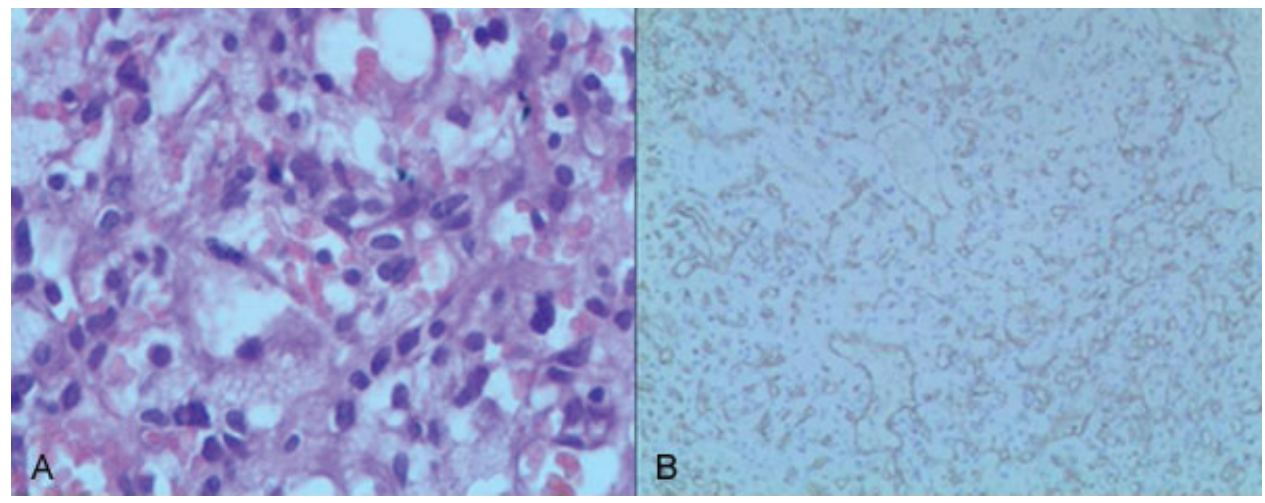

Fig. 3 Histology and immunohistochemistry of a solid hemangioblastoma. (A) A histological picture (H-E, $\times 400)$, showing numerous stromal cells with clear and partly vacuolated cytoplasms and intermingled capillary vessels. No atypical cells were seen in sections examined. (B) An immunohistochemical picture (CD34 staining, $\times 100)$ showing positive stain for CD34.

The clinical presentation of hemangioblastomas usually depends on the anatomical location as well as size of tumor within the CNS. When located in cerebellum, patients may present with signs of cerebellar dysfunction, such as ataxia and discoordination, or with symptoms of increased intracranial pressure due to obstructive hydrocephalus, including headache, nausea, vomiting, and dizziness. ${ }^{6}$ The most com- mon location for hemangioblastoma is the cerebellum, although other unusual locations have also been reported. ${ }^{7,8}$

According to an analysis of MRI manifestations of 18 surgically verified posterior fossa hemangioblastomas, these tumors may appear as a cyst with a small enhancing mural nodule, a solid lesion without a cystic component, or a solid lesion with one or more intratumoral cysts. ${ }^{9} \mathrm{~A}$ cyst with a

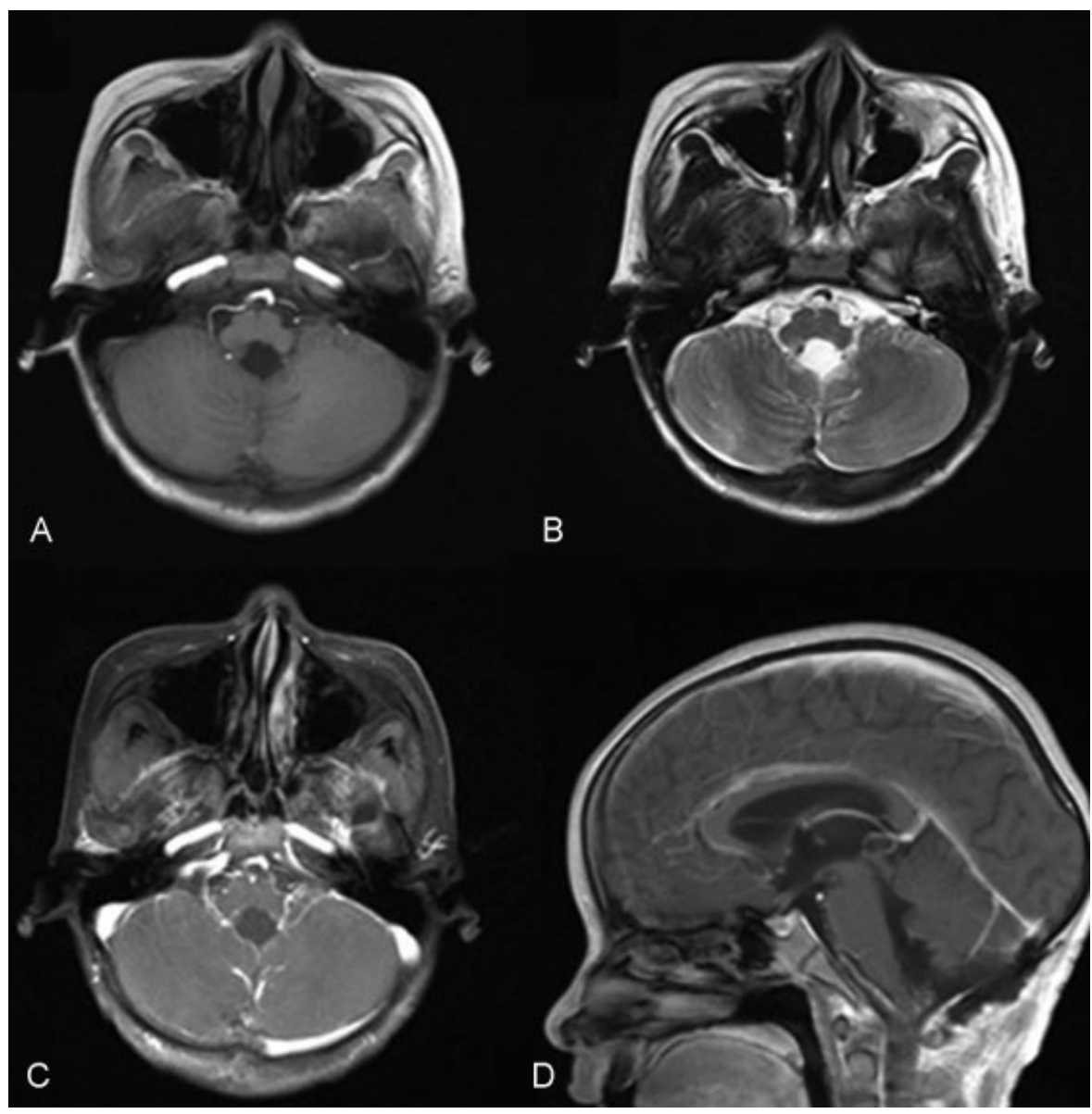

Fig. 4 MR images of the brain 1 week after resection of the tumor. (A) An axial T1-weighted image, (B) an axial T2-weighted image, (C) an axial gadolinium-enhanced image, and (D) a sagittal gadolinium-enhanced image show the total surgical resection of the mass without residual tumor. Note the fourth ventricle remains slightly enlarged. 
small enhancing mural nodule is the most common presentation and solid tumors are relatively uncommon. However, solid hemangioblastomas represent a surgical challenge due to their hypervascularity and their frequent location in functionally important regions of the CNS. ${ }^{10}$ It is therefore vitally important and necessary to obtain as much detailed information of a solid hemangioblastoma as possible, especially for children.

Histopathologically, solid hemangioblastomas are composed of proliferating blood vessels intermingled with stromal cells which are characterized by large nuclei and vacuolated cytoplasm. ${ }^{11}$ Radiologically, solid hemangioblastomas can be characterized properly and noninvasively by neuroimaging techniques such as CT and MRI. At CT scan, these tumors are well-defined solid isodense masses with prominent, nearly homogeneous enhancement. CTA reconstruction can show multiple serpentine vessels, a finding that reflects rich tumor vascularization, which may be useful in surgical planning to avoid intraoperative bleeding and complications. On MRI, these tumors are often hypo- to isointense on T1-weighted images and hyperintense on T2weighted and FLAIR images, with intense enhancement after contrast administration. The feeding or draining vessels in the periphery of the mass may appear as serpentine signal voids, which is an MR characteristic of solid hemangioblastomas. Our case is a solid hemangioblastoma located in the inferior cerebellar vermis in a child. The radiographic features of our case correlate closely with the morphological features of the tumors observed during surgical and histopathological examination.

Radiologically, hemangioblastomas can sometimes be mistaken for meningiomas. CTA and MRI can be of considerable help in identifying vascular anatomy of solid hemangioblastoma, providing an important differentiating factor. Other possible differential diagnostic considerations include medulloblastoma, ependymoma, and arteriovenous malformation (AVM). Medulloblastoma, typically derived from the roof of the fourth ventricle, accounts for 35 to $40 \%$ of all posterior fossa tumors in children with peak occurrence at 4-8 years old. It appears hyperdense relative to brain on CT and heterogeneous signal intensity on T2-weighted images, but without serpentine signal voids within or in the peripheral of the tumor. The enhancement of medulloblastomas may not be as prominent as that of hemangioblastomas. Also, discovery of leptomeningeal or cerebrospinal fluid dissemination of medulloblastomas is helpful in distinguishing the two. ${ }^{12}$ Ependymoma is the third most common posterior fossa tumor in children. Approximately $70 \%$ of intracranial ependymomas are infratentorial and arise from ependymal cells lining the floor of the fourth ventricle and foramen of Luschka. ${ }^{12}$ Inside the tumors, calcifications and areas of cystic degeneration can be seen, therefore producing heterogeneous enhancing patterns. There are no serpentine signal voids in ependymomas. AVMs are uncommon vascular abnormalities in children (1.4-2.8\%) and 90\% of all AVMs are supratentorial. ${ }^{13}$ Thus, infratentorial AVMs of children are even more uncommon. The lesions are irregular with calcification or hemorrhage on $\mathrm{CT}$, and enhancement after contrast administration; MRI shows an inhomogeneous signal void on T1- and T2- weighted images indicating dilated feeding arteries and multiple draining veins, commonly with hemosiderin suggesting prior hemorrhage.$^{14}$ In addition, the focal atrophy in the vicinity of the AVMs is frequently noted ${ }^{15}$ due to ischemia as a result of arteriovenous shunting.

In summary, although solid hemangioblastomas are rare, especially in children, they constitute an important clinical entity because the morbidity and mortality associated with them can be reduced significantly if diagnosed and treated timely and appropriately. ${ }^{16}$ This case draws our attention to a differential diagnosis of solid hemangioblastomas in children when a solid tumor located in the cerebellum is associated with hypervascularity and intense enhancement. The highly vascular nature of the lesions discourages biopsy of the tumor due to the risk of hemorrhage. Imaging alone is usually necessary and sufficient to confirm the diagnosis of hemangioblastoma. CT and MRI can provide detailed information of the tumor as well as adjacent structures, which is of great significance for choosing the proper surgical approach and assessing the risks and prognosis of treatment.

\section{References}

1 Louis DN, Ohgaki H, Wiestler OD, et al. The 2007 WHO classification of tumours of the central nervous system. Acta Neuropathol 2007;114(2):97-109

2 Leung RS, Biswas SV, Duncan M, Rankin S. Imaging features of von Hippel-Lindau disease. Radiographics 2008;28(1):65-79, quiz 323

3 Fisher PG, Tontiplaphol A, Pearlman EM, et al. Childhood cerebellar hemangioblastoma does not predict germline or somatic mutations in the von Hippel-Lindau tumor suppressor gene. Ann Neurol 2002;51(2):257-260

4 Neumann HP, Eggert HR, Weigel K, Friedburg H, Wiestler OD, Schollmeyer P. Hemangioblastomas of the central nervous system. A 10-year study with special reference to von Hippel-Lindau syndrome. J Neurosurg 1989;70(1):24-30

5 Gonc N, Engiz O, Neumann HP, et al. Two pediatric patients with Von Hippel-Lindau disease type 2b: from patient to screening, from screening to patient. J Pediatr Endocrinol Metab 2011;24(12):109-112

6 Lonser RR, Glenn GM, Walther M, et al. von Hippel-Lindau disease. Lancet 2003;361(9374):2059-2067

7 Mills SA, Oh MC, Rutkowski MJ, Sughrue ME, Barani IJ, Parsa AT. Supratentorial hemangioblastoma: clinical features, prognosis, and predictive value of location for von Hippel-Lindau disease. Neuro-oncol 2012;14(8):1097-1104

8 Chen C-Y, Chen P-H, Yao M-S, Chu J-S, Chan WP. MRI of hemangioblastoma in the conus medullaris. Comput Med Imaging Graph 2008;32(1):78-81

9 Lee SR, Sanches J, Mark AS, Dillon WP, Norman D, Newton TH. Posterior fossa hemangioblastomas: MR imaging. Radiology 1989; 171(2):463-468

10 Rachinger J, Buslei R, Prell J, Strauss C. Solid haemangioblastomas of the CNS: a review of 17 consecutive cases. Neurosurg Rev 2009; 32(1):37-47, discussion 47-48

11 Hussein MR. Central nervous system capillary haemangioblastoma: the pathologist's viewpoint. Int J Exp Pathol 2007;88(5):311-324

12 Plaza MJ, Borja MJ, Altman N, Saigal G. Conventional and advanced MRI features of pediatric intracranial tumors: posterior fossa and suprasellar tumors. AJR Am J Roentgenol 2013; 200(5):1115-1124 
24 CT and MRI Features of a Solid Hemangioblastoma Sun et al.

13 Di Rocco C, Tamburrini G, Rollo M. Cerebral arteriovenous malformations in children. Acta Neurochir (Wien) 2000; 142(2):145-156, discussion 156-158

14 Vilanova JC, Barceló J, Smirniotopoulos JG, et al. Hemangioma from head to toe: MR imaging with pathologic correlation. Radiographics 2004;24(2):367-385

15 Ogilvy CS, Stieg PE, Awad I, et al; Stroke Council, American Stroke Association. Recommendations for the management of intracranial arteriovenous malformations: a statement for healthcare professionals from a special writing group of the Stroke Council, American Stroke Association. Circulation 2001; 103(21):2644-2657

16 Vougioukas VI, Gläsker S, Hubbe U, et al. Surgical treatment of hemangioblastomas of the central nervous system in pediatric patients. Childs Nerv Syst 2006;22(9):1149-1153 